\title{
Psychological Factors in Recruitment
}

\author{
Valeriu Deciu \\ University “Alexandru Ioan Cuza”, Iasi, Romania \\ Email: deciuvaleriu@gmail.com
}

How to cite this paper: Deciu, V. (2021). Psychological Factors in Recruitment. Psychology, 12, 1933-1943.

https://doi.org/10.4236/psych.2021.1212117

Received: October 26, 2021

Accepted: December 7, 2021

Published: December 10, 2021

Copyright $\odot 2021$ by author(s) and Scientific Research Publishing Inc. This work is licensed under the Creative Commons Attribution International License (CC BY 4.0).

http://creativecommons.org/licenses/by/4.0/

\begin{abstract}
Evaluating the most appropriate candidate for recruitment is a necessary process that determines an organization's general performance. Therefore, different rules, guidelines, and regulations have been developed to guide evaluating candidates for recruitment. In other words, there are ethical standards that all organizations should adhere to when assessing the most appropriate candidates for recruitment. Since the evaluation of the best candidates for recruitment is a process that involves the interaction of human beings, different psychological aspects are applied during the process. Generally, psychology plays a vital role in all the processes that are carried out in all organizations. These processes include the recruitment of new employees, dispute resolution, and taking disciplinary action. During recruitment, the employer uses psychology to determine the motivation behind the candidate's application, hence helping the employer determine how they should think and behave during recruitment. This research employs a qualitative method of analysis to examine recruitment strategies utilizing three specific psychological factors.
\end{abstract}

\section{Keywords}

Recruitment, Psychological Factors, Recruitment Strategy, Psychological Functioning, Ethics \& Compliance in Recruitment

\section{Introduction}

Different psychological factors affect the process of recruiting employees in an organization (Olšovská \& Švec, 2017). In most industries, there has been a rise in the need to utilize psychological techniques to select candidates. The industrial revolution has also enhanced the use of psychological techniques during recruitment to navigate the barriers associated with the psychological factors that positively impact recruitment. Organizations are expected to implement effective strategies that promote positive psychological factors. During recruitment, employers research the candidates who are psychologically fit for the job vacan- 
cy. It is because different psychological factors affect the performance of an employee. Previous studies indicate that various psychological factors affect workers' mental and general health, affecting their general performance. Employers must use psychological techniques appropriately to determine the best person to recruit. Some of the psychological factors that affect recruitment include personalities, the functioning of the candidate, and their mental state. Since personalities affect an organization's work culture, it is vital to concentrate on the applicants' personalities. Professionalism, energy level, intellectual curiosity, and self-monitoring are among the psychological factors that affect recruitment. They affect the process of recruitment since all employers look for these personality traits in candidates. Also, ethics and compliance policies require employers to look for positive personality traits during recruitment.

The psychological functioning of the candidate is a psychological factor that affects recruitment since the ethical and compliance standards for recruitment require a candidate to have appropriate basic psychological functions such as feeling, thinking, intuition and sensation. Higher psychological functions are other psychological aspects that an individual socially acquires. The employer has to consider the candidates' higher psychological functions before determining the best candidate to recruit. The mental state of the candidates is another psychological factor that affects the process of recruitment. According to the ethics and compliance policies, an employer should recruit a candidate who is mentally well. The recruitment process is significant since it determines the performance of the firm and employees; therefore, this paper analyses the psychological factors that should be considered when evaluating candidates for recruitment.

\section{Methods and Objectives}

\subsection{Materials and Methods}

This research aims to uncover the psychological factors that should be considered when evaluating candidates for recruitment. This study also explains the strategies implemented by the recruiters to measure these psychological factors among the candidates. The research methods applied during a study determine the validity and credibility of the study. The research method used during this study involved analyzing previous research articles regarding the psychological factors considered when evaluating candidates for recruitment.

\subsection{Results and Discussion}

The three main psychological factors that should be considered when evaluating candidates for recruitment are the personality, mental state, and psychological functions of the candidates. These psychological factors affect recruitment since it determines the process of selecting the most qualified candidates for a vacant job position. Assessing candidates' mental and psychological state is necessary during recruitment since it determines the organization's performance. 


\section{Applying Psychological Factors}

\subsection{The Personality of the Candidate}

According to Cole et al. (2009), recruiters look for a candidate whose personality matches the job vacancy and the organization at large, indicating that personality influences job performance. The study was conducted to examine whether the job provided on social networking sites such as LinkedIn can be used by employers to determine the personality traits of an applicant. The study was conducted to determine if personality traits indicated by the profile owner on social networks correlate with the self-related personality traits of the profile's owner. The results from the study indicated that personality traits posted by the profile owner on social networks allowed recruiters to gain better inferences of their traits, such as self-presentation and extraversion.

Bansal, Bhushan, \& Gupta (2020) claim that personality traits greatly affect work management. Personality traits are defined as the static perceptual structure, how a person thinks, behaves, or feels. For instance, extraversion is a personality trait associated with assertiveness, talkativeness, gregariousness-people with this personality trait are expressive, active, enthusiastic, and energetic. Specifically, four of the big five personalities (agreeableness, extraversion, openness, and conscientiousness) positively correlate with job engagement. Candidates with positive personality traits uphold work engagement in an organization compared to those with negative personality traits.

Organizations look for candidates with the right qualifications and personality traits. Recruiting candidates whose personality traits fit with the company's requirements enhances turnover intentions and job satisfaction. For this reason, most organizations use personality tests to determine the candidates' personality traits after screening the candidates. However, the high cost of conducting personality tests is another factor that has made it hard for most organizations to determine the personality traits of applicants appropriately. Most companies use resumes and application letters to determine the candidates' personality traits in the pre-selection stage of applicant's personality traits, and application letters provide valid cues that infer their personality candidates' personality traits and application letters. The rise in the use of modern technology and the internet has made it easy for recruiters to assess candidates' personality traits through the profiles they have created on social networks such as Facebook. Such profiles may also be utilized to forecast the job output of the applicant.

Since personality traits are a relevant factor to be considered during recruitment, many recruiters have put more effort into ensuring the candidates' personality traits are well established. For instance, it has been revealed that using social network profiles to determine a candidate's personality traits is more effective than using job-related social network profiles. Identifying the right personality traits for a candidate is important since it allows the recruiters to identify the candidate's behavioral tendencies, which enhances the understanding of their performance concerning the organization's culture. For instance, profes- 
sionalism is one of the personality traits that is considered during the evaluation of candidates. Recruiting candidates with a high level of professionalism is a critical factor that helps in upholding employee retention.

Professionalism plays a vital role in all areas of an organization, especially recruitment. Professionalism is defined as an individual's behavior, conduct, and attitude at work. Professionalism is one of the personality traits that affect recruitment since recruiters look for candidates who portray the highest level of professionalism during recruitment. A high level of professionalism leads to a high level of work excellence and ethics, workplace success, and a strong professional reputation. One factor that recruiters consider in determining a candidate's professionalism is their time management, dressing, communication, and resilience mode. All companies look for workers who maintain professionalism since these workers represent the company. Therefore, any good or bad action they perform affects them and the company.

Aven \& Alm (2020) state in the article that employers are no longer interested in a candidate's expertise during recruitment, but they also focus on other personal qualities such as professionalism. In the article, social trustee' professionalism insists that candidates express or embody broader social values that enable them to be more productive at work. Hybrid professionalism and organizational professionalism theories state that the rise in market competition has increased the need for professionalism among the employees since they enhance public accountability, commercialism, and managerialism. Therefore, the impact of professionalism on the performance of organizations has made professionalism a psychological factor that affects the recruitment process.

A candidate's level of energy determines their productivity and ability to work. This forces the recruiters to look for candidates with high positive energy during recruitment. Thus, the level of energy is another psychological factor that recruiters consider during recruitment. During recruitment, employers do not only look at the candidates' level of experience, but they also consider their energy levels by identifying the interests of the candidates and the activities they participate in outside work. Recruiting candidates with high energy levels is important since it ensures that the organization employs highly motivated workers. Additionally, high energy levels determine a candidate's level of self-confidence, attitude, and problem-solving skills in the workplace. The recruiters distinguish between energetic candidates and other candidates by observing their commitment and passion.

Using interviews is the best way of determining energetic candidates during recruitment. Interviews help identify energetic candidates since they can describe their long-term plans and goals with enthusiasm, proving that they are determined to achieve these goals. The techniques, activities, and methods applied by the candidate to reach their goals also tell about their energy levels. The way a candidate describes their strategies on overcoming barriers towards their goals and capitalizes on available opportunities also tells about their energy le- 
vels. During an interview, the candidate's creative and innovative responses determine their professional and personal levels, indicating their energy levels.

Candidates' problem-solving skills also indicate their energy level since highly energetic employees have gone through difficult situations and came out successfully. Recruiting energetic employees who can effectively be cool under fire is appropriate for an organization since problems occur inevitably in all organizations. It is important to investigate the candidates' past performance to determine their energy levels by inquiring on the number of times they have completed a daily assignment that took extra work that resulted in the acquisition of new skills. After working to take new tasks, people who use their extra time have more commitment and energy levels than those who wait to be instructed by their supervisor.

Self-improvement strategies applied by a candidate, such as the desire to improve workplace conditions, are related to the energetic behaviors of employees in the workplace. The actions performed by energetic employees to improve the conditions at work include reducing errors, costs, and wastes in the workplace. An example of an energetic candidate is a candidate who can describe the things they have completed in the past to further their careers. Their loyalty and commitment also determine a candidate's energy level since energetic workers can meet tight deadlines. In some situations, they work for long hours to meet the expectations of all the stakeholders, and this describes their energy levels and desire to meet difficult goals under adverse conditions. The ability to work appropriately with other people in the organization also describes the energy levels of candidates.

Another psychological factor to be considered in evaluating the best candidate is the candidate's intellectual curiosity. Intellectual curiosity is a form of curiosity that is associated with the acquisition of knowledge. Recruiters seek intellectually curious candidates since they have an innovative approach to solving the problems in an organization. They are also more flexible because they can adapt to the changes that might occur in an organization. The changes in the climate of the job market have increased the need for organizations to recruit intellectually curious candidates since they are fast learners who can think critically in various conditions. The knowledge they also possess positively affects other people who listen attentively to them, thereby encouraging knowledge and information sharing. They bring knowledge to the organization due to their constant learning. They also fight the status quo by discovering unchartered territories. Intellectually curious employees also collaborate effectively with other workers in the organization for effective performance.

Self-monitoring among the candidates is a factor that is considered when selecting the best candidates for recruitment. According to Nwanzu \& Babalola (2019), self-monitoring predicts a candidate's attitude towards organizational change. This study was conducted to determine whether self-monitoring, self-efficacy, and optimism could predict the attitude towards organizational change. The results 
from the study indicated that self-monitoring, self-efficacy, and optimism positively predict a person's attitude towards organizational change. Organizations select candidates with self-monitoring traits since they can positively contribute to organizational changes. Therefore, the self-monitoring trait is another psychological factor that is considered while recruiting new employees. Most organizations have implemented rules, policies, and strategies for the recruitment of candidates with self-monitoring traits.

\subsection{The Mental State of the Candidate}

The mental state of the candidate is another psychological factor that affects recruitment since recruiters are more interested in workers with the right mental or psychological abilities for their job. For this reason, most organizations conduct psychological assessments during recruitment to help recruiters focus on both the positive and negative differences that a candidate can make on the job they will be performing, the teams they will be working with, and the organization at large. These tests determine the candidate's mental state by determining the candidate's emotional quotient and intelligence. The EQ factor and intelligence of the candidate are expected to match with the benchmarked percentage attribute to the vacant position. The failure to assess the personality and cognitive traits leads to a loss of effort and time. The behavior and cognitive functioning of an individual determine their mental state. Other different aspects of mental health determine the mental state of a candidate. These aspects include the candidate's behavior, general appearance, thought and perception, attitude and insight, mood and affect, and cognitive abilities.

A candidate's physical behavior is a psychological factor that affects the recruitment process since recruiters should select candidates with appropriate physical behaviors. Physical behaviors also play an important role during physical communication. For instance, the body language of an individual tells a lot about their mental state and personality. Maintaining eye meaningful eye contact and appropriate body movements tells whether a candidate understands whatever they are asked. A candidate who understands all the information about the organization and the vacant job position is said to be mentally well. Therefore, recruiters use interviews to ask the candidates relevant questions about their mental or psychological state.

The thoughts and perception of the candidate are other psychological factors that are considered during the recruitment process. These two aspects of mental health help the recruiter to assess the mental state of the candidate. Thought processing is determined by how the candidate expresses their thoughts. According to Voss \& Das (2020), the thoughts of people with normal thought processes are described to be goal-directed and linear. On the other hand, irregular thought processes are described as circumstantial since they are associated with the flights of ideas, thought blocking, and perseveration. The way people process their thoughts determines their mental state, general productivity, and 
performance at work. The candidate's perception, especially towards the job, helps the recruiters determine their mental state. Most employers recruit candidates whose behaviors indicate they have a positive perspective towards the company, its activities, and the vacant position. The perception of the candidate is a factor that determines employee motivation and general performance.

Recruiters also consider mood and affect when selecting candidates during recruitment. Affect refers to the candidate's immediate expression of their emotions, while the mood is the sustained emotional make-up of the candidate's personality. People who have mental illnesses also experience mood disorders. Candidates with mood disorders are not preferred during recruitment since these conditions negatively affect how a person relates with others in the organization, undermining their general productivity and performance. Workers with mood disorders experience difficulty concentrating, making it hard for them to complete urgent tasks on time. Also, difficulty in concentration may lead to errors and mistakes at work.

On the other hand, employers look for candidates with positive affectivity during recruitment. Workers with positive affectivity have positive expressions and emotions such as pride, cheerfulness, enthusiasm, joy, and energy. Negative affectivity refers to negative expressions and emotions that include disgust, sadness, fear, lethargy, and distress. Both negative and positive affectivity plays a vital role in our enjoyment and daily experience. A candidate's affectivity influences their opinions, performance, brain activity, and abilities. In addition, negative and positive affectivity has an impact on the personal traits of an individual.

A study by Wall, Russell, \& Moore (2017) indicates that positive emotions such as positive affectivity positively impact organizations, individuals, and society. Importantly, it contributes to broader health, wellbeing, resilience, and job satisfaction among the employees of an organization. A candidate with positive emotions can explore their values, accomplishments, meaning, and vision of the organization both on a personal and organizational level. Since learning is an important process for all organizations, people who are more positively engaged emotionally facilitate workplace learning. Positive emotions stimulate knowledge-sharing processes and shape specific cognitive contents and sense in a particular situation, for instance, negative emotions or affectivity such as anger, anxiety, fear, or hope influence a person's ability to engage in sensible activities. However, different factors affect the emotion of candidates. Such factors include gender, race, or class.

The candidate's cognitive abilities are a psychological factor that determines the selection of the best candidate during recruitment. Cognitive ability is defined as the brain's occurring skills used in both complex and simple tasks. The cognitive abilities include memory, attention, logic, and reasoning. Attention is the candidate's ability to perform tasks while staying focused for a long time without being distracted or performing multiple tasks simultaneously. It determines how an individual can perform their delegated tasks or duties. Memory is 
the ability to retain past or recent information. Retaining past information is regarded as long-term memory while retaining recent information is regarded as short memory. Logic and reasoning refer to the cognitive strengths that enable the candidate to assess a problem and appropriately find the best solution. Other cognitive tasks such as visualizing solutions and understanding symbols work in conjunction with auditory and visual processing to speed up information processing.

The ethics and compliance policies require all recruiters to find candidates with cognitive skills and specific aptitudes that best match the vacant job. Aptitude refers to the combination of cognitive abilities that indicates a person's ability to perform certain tasks appropriately and pick up new skills while working. Recruiters use different strategies to determine a candidate's cognitive abilities. One strategy is to determine the candidate's experience and past performance to determine whether they managed to use their cognitive skills appropriately to gain more experience, skills, and knowledge. All workers need to possess appropriate cognitive abilities since it enables them to analyze the resources in the organization by analyzing the provided data. It also enables the employee to identify the patterns in the organization for effective strategic decision-making. Therefore, recruiters are forced to consider the candidates' cognitive abilities, making it one of the psychological factors to be considered when recruiting candidates.

\subsection{The Psychological Functioning of the Candidate}

The psychological functioning of the candidate is another psychological factor that determines the selection of a candidate during recruitment. Olšovská \& Švec (2017) state that determining a candidate's psychological functioning entails testing all the psychological functions that include creativity, memory, other abilities such as talent and communication. According to the ethical standards for all organizations, recruiters are expected to hire creative employees who can build better teamwork. Notably, the creativity of a candidate is a psychological factor that affects the process of recruitment.

A study by Casey (2020) indicates that there is a connection between collaboration and creativity. The research was founded on an analysis of personal writing samples ranging from 2007 to 2017. The article proves that collaboration among the writers was associated with a high level of creativity compared to one person. Similarly, organizations recognize the important role played by creative employees towards teamwork and collaboration in the organization. Similarly, collaboration among the employees improves creativity among the team members since it ensures that they relate positively to effective knowledge sharing. In other words, collaboration increases creativity through the effective sharing of knowledge and ideas. In essence, creative and collaborative employees are more innovative since they bring in new news. Implementing the ideas from any stakeholder also requires a lot of collaboration and creativity to save on time and cost. 
Recruiters use interview questions to assess the creativity levels of candidates. They use behavioral job interview questions to determine whether you have encountered a situation that required creativity in the past. The candidate is expect4ed to be creative while answering such questions during an interview since the employers keenly observe how the candidate provides the answers. The observations made by the recruiter tell about the candidate's personality, ethics, and thought processes. Thus, it shows if the candidate is creative or not. However, recruiters have found it hard to measure the current level of creativity for an individual candidate since everyone is creative differently.

Candidates with other unique abilities such as talents are viewed to have a higher level of creativity. Nuhoglu \& Akgül's (2019) study to determine the relationship between talent and creativity indicates that talented students are more creative since they portray effective problem-solving skills and fluency. The students involved in the study also revealed that talent portrays creativity since it increases flexibility among the students when sharing ideas by enabling them to share many ideas or opinions in different categories. The important role played by talent has led to the establishment of talent management programs in most organizations. Talent management refers to the continuous process of attracting, hiring, developing, motivating, promoting, and retaining the most talented workers in the organization to achieve long-term and short-term goals. It also ensures that operational efficiency is enhanced for profit maximization. Hiring the most talented candidates during recruitment is part of talent management to ensure that more creative and talented workers are employed. However, talent management involves recruiting talented employees. It also involves exploring the hidden qualities of workers to develop and nurture them to best suit the company's needs and objectives. Thus, recruiters also prefer candidates with unique talents and abilities since they are more creative and productive.

The candidate's ability to communicate both through verbal and non-verbal communication is a psychological factor that is considered when during recruitment. Recruiters look for candidates with effective communication skills since it affects their relationship with other employees, hence determining their general performance. Candidates who portray effective communication skills are more productive since they can facilitate knowledge sharing and effective communication among other employees in the organization. Effective communication applies to all candidates, including those who do not qualify for the vacant position, since it determines the candidate's reaction who did not qualify for the job. That is to say, such candidates will appropriately use their communication skills to maintain a positive experience of the company even if they did not get the job.

Communication is part of everyday human life since all human beings communicate with each other more frequently. The way a candidate communicates during recruitment helps the recruiter to identify the person's character or trait. It also tells about their level of creativity in terms of how they present their arguments, opinions, or ideas. Recruiters consider communication an important 
factor during recruitment since all workers in an organization represent their organization outside. Therefore, they should all possess effective communication skills that enable them to represent the organization positively. Generally, effective communication enhances business communication, which refers to the interaction between the employees inside and outside the organization.

Dwyer \& Hopwood (2019) assert that business communication among all employees ensure that all the necessary activities and operation in an organization are carried out smoothly and effectively. Formal business communication manages the interaction between all the employees for the achievement of organizational goals. Despite the importance of effective communication in the organization, there are many challenges faced by the business organization that prevents effective communication. As such, all business organizations need to implement effective strategies that enhance the management of business communication. Organizational culture is used to promote effective communication among the workers in an organization. Establishing an organizational culture that upholds effective communication makes it possible for a newly employed candidate to adapt easily to the organization's cultures, especially when it comes to communication. Ultimately, effective communication will enhance teamwork and collaboration to improve the organization's general performance.

\section{Conclusion}

Evaluating the most appropriate candidate for recruitment is a significant process that determines the organization's general performance. Different psychological factors affect recruiting employees in an organization-the process of recruiting the most qualified candidates. During recruitment, employers research the candidates who are psychologically fit for the job vacancy. The psychological functioning of the candidate is a psychological factor that affects recruitment since the ethical and compliance standards for recruitment require a candidate to have appropriate basic psychological functions such as feeling, thinking, intuition and sensation. An individual's personality determines their ability to relate well with other people around them, especially in a workplace setting. Generally, various psychological factors affect the way people think or relate.

The three main psychological factors that should be considered when evaluating candidates for recruitment are the personality, the mental state, and the psychological functions of the candidates. These psychological factors considered by employers during recruitment help determine the general performance and productivity of the workers. According to Cole et al. (2009), recruiters look for a candidate whose personality matches the job vacancy and the organization at large, indicating that personality impacts job performance. Also, the mental state of the candidate is another psychological factor that affects recruitment since recruiters are more interested in workers with the right mental or psychological abilities for their job. The psychological functioning of the candidate is 
another psychological factor that determines the selection of a candidate during recruitment. Therefore, organizations should determine the best way to ensure that all the psychological factors that impact recruitment positively impact the organization. In other words, the negative impacts associated with these psychological factors should be minimized while the positive impacts should be maximized.

\section{Conflicts of Interest}

The author declares no conflicts of interest regarding the publication of this paper.

\section{References}

Aven, B. H., \& Alm-Andreassen, T. (2020). Trustee Professionalism Transformed: Recruiting Committed Professionals. Current Sociology.

https://doi.org/10.1177/0011392120969759

Bansal, E., Bhushan, P., \& Gupta, Y. (2020). Personality Traits and Work Engagement: A Case Study on Female Bank Employees in Banking Sector. Parikalpana: KIIT Journal of Management, 16, 72-83.

Casey, R. (2020). Creativity and Collaboration: The Relationship of Fact and Fiction in Personal Writing. The Macksey Journal, 1, 21726.

Cole, M. S., Feild, H. S., Giles, W. F., \& Harris, S. G. (2009). Recruiters' Inferences of Applicant Personality Based on Resume Screening: Do Paper People Have a Personality? Journal of Business and Psychology, 24, 5-18. https://doi.org/10.1007/s10869-008-9086-9

Dwyer, J., \& Hopwood, N. (2019). The Business Communication Handbook. Cengage AU.

Nuhoglu, H., \& Akgül, S. (2019). Analysis of the Relation between Creativity Level and Problem-Solving Skills of Gifted and Talented Students. Educational Research and Reviews, 14, 518-532. https://doi.org/10.5897/ERR2019.3790

Nwanzu, C. L., \& Babalola, S. S. (2019). Examining Psychological Capital of Optimism, Self-Efficacy, and Self-Monitoring as Predictors of Attitude towards Organizational Change. International Journal of Engineering Business Management, 11. https://doi.org/10.1177/1847979019827149

Olšovská, A., \& Švec, M. (2017). Use of Psychological Examinations of Employees and Job Applicants in Personnel Management. Issues of Human Resource Management, 23-41. https://doi.org/10.5772/67868

Voss, R., \& Das, J. M. (2020). Mental Status Examination. StatPearls.

Wall, T., Russell, J., \& Moore, N. (2017). Positive Emotion in Workplace Impact: The Case of a Work-Based Learning Project Utilizing Appreciative Inquiry. Journal of Work-Applied Management, 9, 129-146. https://doi.org/10.1108/JWAM-07-2017-0017 